\title{
ON THE ORDER OF EXPONENTIAL GROWTH OF THE SOLUTION OF THE LINEAR DIFFERENCE EQUATION WITH PERIODIC COEFFICIENT IN BANACH SPACE
}

\author{
H. ATTIA HUSSEIN \\ Department of Mathematics \\ Faculty of Science \\ Alexandria University \\ Alexandria, Egypt \\ (Received May 11, 1984)
}

ABSTRACT. An equation of the form $y-A(t) y=f(t)$ is considered, where $\Delta y=\frac{y(t+\delta)-y(t)}{\delta}$, and the necessary and sufficient criteria for the exponential growth of the solution of this equation is obtained.

KEY WORDS AND PHRASES. Difference equations, solution of exponential growth. 1980 MATHEMATICS SUBJECT CLASSIFICATION CODE. $39 A 10$.

1. INTRODUCTION.

Let $E$ be a complex Banach space. Denote by $\{A(t): t \geq 0\}$ a family of linear bounded operators from $E$ into itself. We assume that $A(t)$ is periodic and strongly continuous in $t \in[0, \infty)$.

Let $\|\cdot\|$ be the norm in $E$. Denote by $E_{\alpha}$ the set of all elements $f(t) \varepsilon E$ such that

$$
\sup \|f(t)\| \exp (-\alpha t)<\infty \text {. }
$$

2. RESULTS.

Let $\Delta y=\frac{y(t+\delta)-y(t)}{\delta}, \delta>0, y(t)$ be a solution of the difference equation

$$
\Delta y-A(t) y=f(t) \quad, t \geq \delta
$$

such that

$$
y(t)=\theta \quad, \quad 0 \leq t<\delta
$$

where $\theta$ is the zero of $E$.

Let us assume that $f \in E_{\alpha}$. The solution of equation (2.1) can be written in the 
form

$$
y(t)=\delta \sum_{i=0}^{t-\delta} A(i) y(i)+\delta \sum_{i=0}^{t-\delta} f(i)
$$

where $t=[n \delta],[a]$ denotes the greatest positive integer $\leq a$ and $\delta$ is a positive integer.

Without loss of generality we suppose that $\delta=1$.

Putting $t=1,2, \ldots, n$ in (2.3), one obtains

$$
y(t)=\sum_{j=1}^{n-1} \prod_{i=n-1}^{j}(I+A(i)) f(j-1)+f(t-1)
$$

where $I$ is the unit operator. Let $w$ be the period of $A(t)$.

$$
\left[\prod_{i=n-1}^{j} B(i)=B(n-1) B(n-2) \ldots B(j), j \leq n-1\right]
$$

Substituting $t=[S w]$ into equation (2.4), we obtain

$$
\begin{aligned}
& y(t)=\sum_{r=1}^{s}\left[\begin{array}{l}
0 \\
\prod=w-1 \\
\text { where }
\end{array}\right. \\
& \quad f_{1}(\xi w)=A(w-1) A(w-2) \ldots A(1) \quad f(\xi w) \\
& \quad f_{2}(\xi w+1)=A(w-1) A(w-2) \ldots A(2) \quad f(\xi w+1) \\
& \quad \ldots \\
& \quad \ldots \\
& \quad f_{w-1}^{w-1}(\xi w+w-2)=A(w-1) f(\xi w+w-2) .
\end{aligned}
$$

Setting $B=\prod_{k=W-1}^{0}[I+A(k)]$ in (2.5) we get

$$
y(t)=\sum_{r=1}^{\frac{t}{w}} B^{\frac{t-r w}{w}}\left\{\sum_{j=1}^{w-1} f_{j}((r-1) w+j-1)+f((r-1) w+w-1)\right\} .
$$

The last equation can be written in the form

$$
y(t)=-\frac{1}{2 \pi i} \oint_{\gamma} \sum_{r=1}^{\frac{t}{w}} \lambda^{\frac{t-r w}{w}}(B-\lambda I)^{-1}\left\{\sum_{j=1}^{w-1} f_{j}((r-1) w+j-1)+f((r-1) w+w-1)\right\}
$$

where $\gamma$ is a contour which circumscribes all the specter of the operator $B,[1]$.

It can be seen that if $f \in E_{\alpha}$, then $(B-\lambda I)^{-1} f \in E_{\alpha}$ for every $\lambda \varepsilon \gamma$. From equation (2.6) we obtain a necessary and sufficient criterion for the exponential growth of the solution with an index $B$. Let $\sigma_{B}$ denote the specter of the operator $B$. Assume that $\lambda_{0} \varepsilon \sigma_{B}$. Set $\alpha_{0}=\frac{1}{W} \ln \left|\lambda_{0}\right|$.

The following theorem holds:

THEOREM. If $f \in E_{\alpha}$, then the solution $y$ of equation (2.1) belongs to $E_{\beta}$ such that 


$$
\begin{aligned}
& \beta=\alpha, \text { when } \alpha>\alpha_{0} \\
& \beta>\alpha, \text { when } \alpha=\alpha_{0} \\
& \beta=\alpha_{0}, \text { when } \alpha<\alpha_{0} .
\end{aligned}
$$

PROOF. To prove the sufficiency, we consider the following three cases:

(1) If $\alpha>\frac{1}{w} \ln |\lambda|$ then $y(t)$ defined by (2.6) belongs to $E_{\alpha}$.

(2) If $\alpha>\frac{1}{w} \ln |\lambda|$ then from (2.6) we obtain

$\|y\| \leq D \sum_{r=1}^{\frac{t}{w}} \exp \left(\frac{1}{w} \ln |\lambda|(t-r w)\right) \sum_{j=1}^{w-1}\left\|f_{j}((r-1) w+j-1)\right\|$

$$
\begin{aligned}
& <D_{1} \exp (\alpha t) \cdot \frac{t}{w}+D_{2} \exp (\alpha t) \cdot(w-2) \frac{t}{w} \\
& <D^{\prime} \exp (\alpha t) \cdot t
\end{aligned}
$$

(where $D, D_{1}, D_{2}$ and $D^{\prime}$ are constants).

This means that $y \in E_{B}$ where $\beta>\alpha$.

(3) If $\alpha<\frac{1}{w} \ln |\lambda|$ and ||$f|| \leq c \exp (\alpha t)$, then from (2.6) we have

$$
\begin{aligned}
& || y|| \leq C_{1} \exp \left(\frac{1}{w} \ln |\lambda| . t\right) \\
& \text { and } y \in E_{\frac{1}{w}} \ln |\lambda| \\
& \left(\alpha<\frac{1}{w} \ln |\lambda|\right) .
\end{aligned}
$$

We now prove the necessity:

If $\lambda_{0}$ is an eigenvalue anci $x_{0}$ is an eigenvector for the operator $B$ such that $B x_{0}=\lambda_{0} x_{0}$

where $x_{0}$ is an element of Banach space such that $\left\|x_{0}\right\|=1$, by taking $f(t)=\exp (\alpha t) \cdot x_{0}$ equation (2.6) with

$(B-\lambda I)^{-1} x_{0}=\frac{x_{0}}{\lambda_{0}-\lambda}$ becomes

$$
y(t)=\sum_{r=1}^{\frac{t}{w}} \exp \left(\frac{1}{w} \ln \left|\lambda_{0}\right|(t-r w)\right)\left\{\sum_{j=1}^{w-1} f_{j}((r-1) w+j-1)+f((r-1) w+w-1)\right\} .
$$

Multiplying the last equation by $\exp \left(-\alpha_{0} t\right)$, where $\alpha_{0}=\frac{1}{w} \ln \left|\lambda_{0}\right|$, we have

$\left.y(t) \exp \left(-\alpha_{0} t\right)=\exp \left(\frac{i \theta t}{w}\right) \sum_{r=1}^{\frac{t}{w}} \exp \left(-\alpha_{0} w r\right)\left\{\sum_{j=1}^{w-1} f_{j}((r-1) w+j-1)+f(r-1) w+w-1\right)\right\}$

where $\theta=\arg \lambda$.

$$
\begin{aligned}
y(t) \exp \left(-\alpha_{0} t\right) & =\exp \left(\frac{i \theta t}{w}+w(1-\alpha)-1\right) \sum_{r-1}^{\frac{t}{w}} \exp \left(\left(\alpha-\alpha_{0}\right) w r\right) \cdot x_{0} \\
& +\exp \left(\frac{i \theta t}{w}\right) \underset{r-1}{\frac{t}{w}} \underset{j-1}{w-1} \exp \left(-\alpha_{0} w r\right) f_{j}((r-1) w+j-1)
\end{aligned}
$$




$$
\begin{aligned}
& =\frac{\exp \left(\frac{i \theta t}{w}+\left(\alpha-\alpha_{0}\right)\right.}{\exp \left(\alpha-\alpha_{0}\right)-1}\left[\exp \left(\alpha-\alpha_{0}\right) t-1\right] x_{0} \\
& +\exp \left(\frac{i \theta t}{w}\right) \sum_{r=1}^{\frac{t}{w}} \sum_{j=1}^{w-1} \exp \left(-\alpha_{0} w r\right) \cdot f_{j}((r-1) w+j-1)
\end{aligned}
$$

Now for the last relation we have the following cases:

1) If $\alpha>\alpha_{0}$ then by using formula (2.8) we get

$$
\lim y(t) \exp \left(-\alpha_{0} t\right)=\infty \text {. }
$$

$t \rightarrow \infty$

This means that $y \notin E_{\alpha_{0}}$ but $y \in E_{\alpha}\left(\alpha>\alpha_{0}\right)$.

2) If $\alpha=\alpha_{0}$ then from (2.8)

$$
\begin{aligned}
y(t) \exp \left(-\alpha_{0} t\right)= & \exp \left(w(1-\alpha)-1+\frac{i \theta t}{w}\right)\left(\frac{t}{w}-1\right) x_{0} \\
& +\exp \left(\frac{i \theta t}{w}\right) \sum_{r=1}^{\frac{t}{w}} \sum_{j=1}^{w-1} \exp \left(-\alpha_{0} w r\right) f_{j}((r-1) w+j-1) .
\end{aligned}
$$

Using the last equation we get

$$
\lim _{t \rightarrow \infty} y(t) \exp \left(-\alpha_{0} t\right)=\infty .
$$

This means that $y \in E \alpha$ but $y \in E_{\beta}(\beta>\alpha)$.

3) If $\alpha=\alpha_{0}$ then from (2.8) we have $y \notin E_{\alpha}$ but $y \in E_{\alpha_{0}}$. This completes the proof.

\section{ACKNOWLEDGEMENT}

The author would like to express his sincerest thanks to Professor Dr. Mahmoud M. El-Borai for his advice during the preparation of this paper.

\section{REFERENCES}

1. HUSSEIN, H. A. On the Bounded Solution of Certain Linear Equations in Partial Differences Equations, J. Natur. Sci. Math. 21, (1981) 165-169.

2. HUSSEIN, H. A. Estimation of the Exponential Growth of the Solution of Certain Linear Partial Differential Equations with a Highest Order Term, (Russian) Differencial' nye Uravnenija 12 (1976) 2279-2280. 


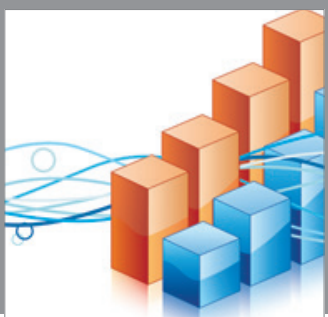

Advances in

Operations Research

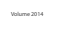

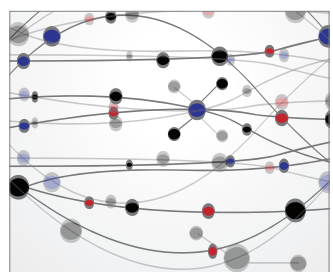

\section{The Scientific} World Journal
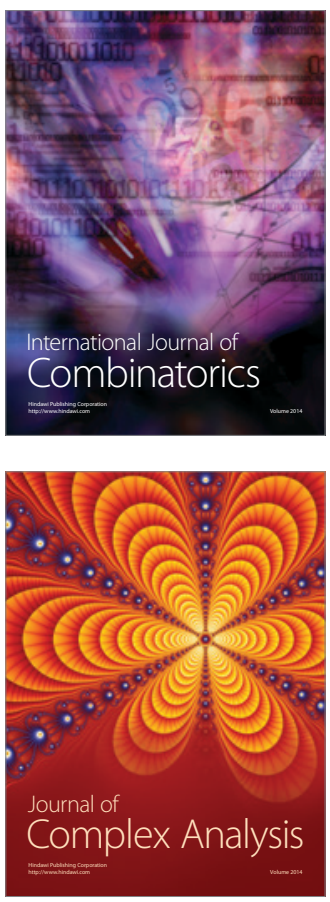

International Journal of

Mathematics and

Mathematical

Sciences
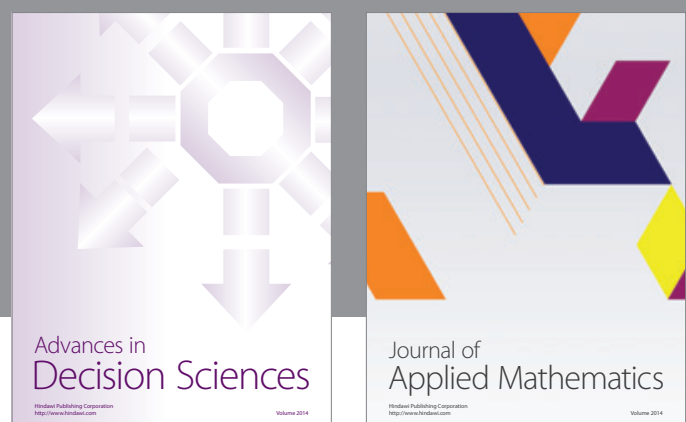

Journal of

Applied Mathematics
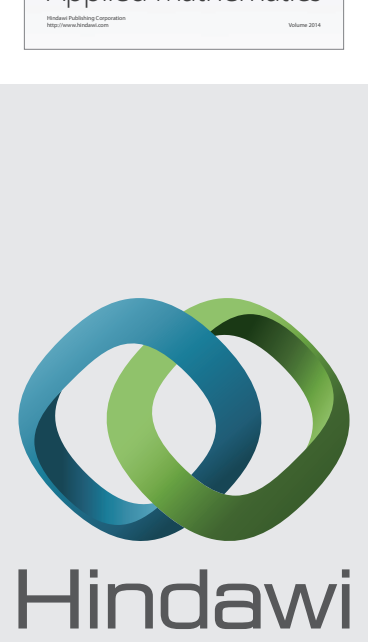

Submit your manuscripts at http://www.hindawi.com
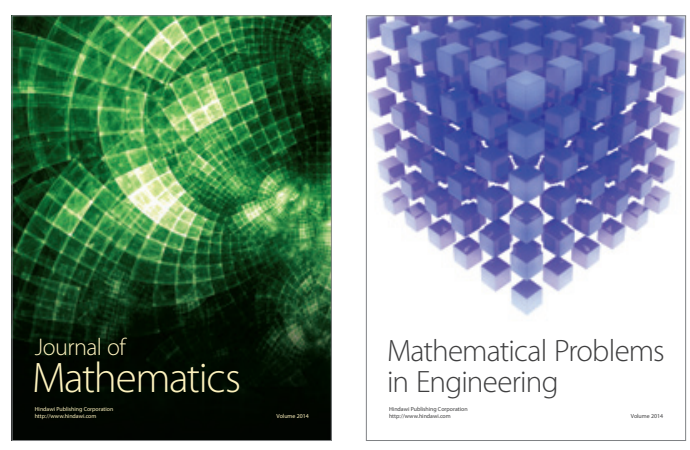

Mathematical Problems in Engineering
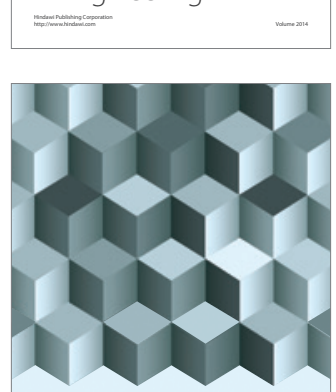

Journal of

Function Spaces
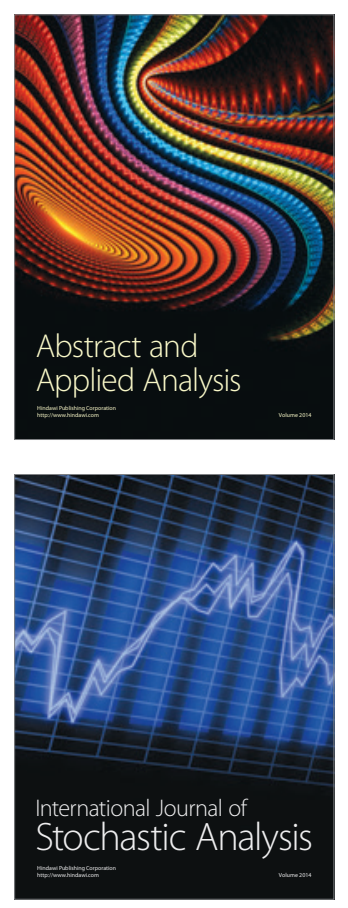

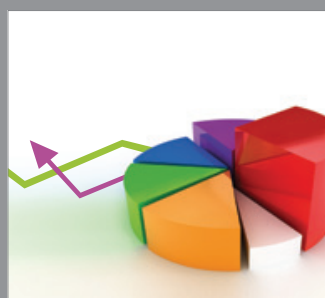

ournal of

Probability and Statistics

Promensencen
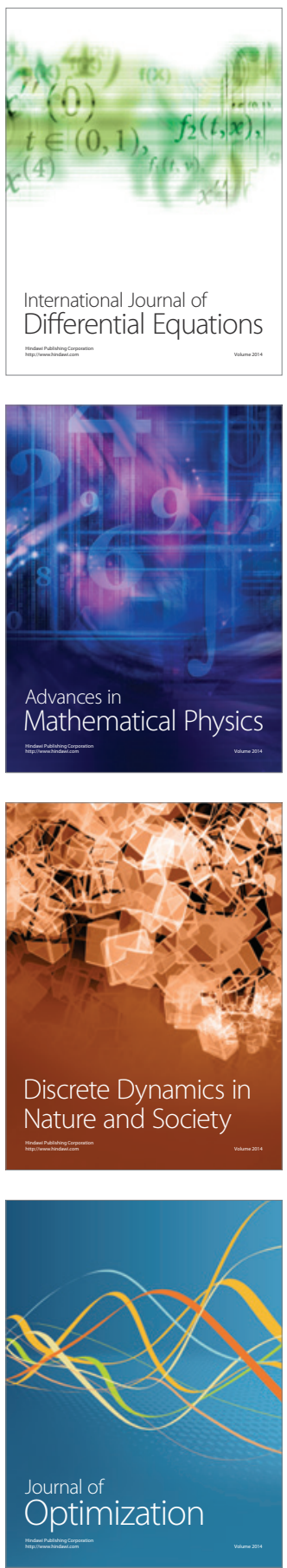